\title{
REORGANIZACIÓN FORMAL Y FUNCIÓN SOCIAL EN UNA LENGUA MINORITARIA: UN EJEMPLO DEL CONTACTO GALLEGO-CASTELLANO
}

\section{InTRODUCCIÓN}

Dentro de los contextos de contacto interlingüístico es posible identificar variados ejemplos de rasgos lingüísticos que, por su ausencia o por su presencia, derivan de la convivencia prolongada y más o menos intensa entre una variedad tipificada como autóctona o nativa, y otra definida como altóctona o foránea.

La investigación sobre los procesos de cambio lingüístico inducido y sobre los fenómenos de interferencia lingüística ha aportado una gran cantidad de material empírico que ejemplifica cuáles son los resultados formales y funcionales derivados del contacto $^{1}$.

Más concretamente, han sido explorados aquellos resultados lingüísticos con respecto a la variedad nativa en el contacto, y sobre los grupos de hablantes autóctonos, en la medida en que la variedad nativa deviene socialmente minoritaria y lingüísticamente recesiva.

Resulta interesante observar cómo, con diversos matices, existe cierta concordancia a la hora de considerar los resultados derivados de los fenómenos de cambio inducido y de interferencia espontánea en el habla. Sobre tales efectos formales y funcionales, dos han sido los ejes de reflexión planteados:

1. Los cambios formales producidos en la variedad nativa conllevan un alto grado de ruptura estructural allí donde se producen, en el sentido de afectar seriamente a los recursos estructurales nativos, bien por simplificación o regularización, bien por pérdida, bien por sustitución foránea, etc. Este efecto de desmembración interna es argüido por cuanto se establece que el

1 Thomason \& Kaufman 1988; Mougeon \& Beniak 1991. 
cambio inducido, en oposición al cambio lingüístico espontáneo, no genera compensaciones internas ${ }^{2}$, y por cuanto el material nativo pasa por un proceso general y profundo de regularización formal, con los consiguientes efectos depredadores sobre los recursos gramaticales y funcionales autóctonos ${ }^{3}$.

En esta misma línea, se ha señalado que la variedad nativa se desmembra formalmente en la medida en que los cambios producidos tiendan hacia la semejanza estructural $y / o$ funcional con la variedad origen (o espejo) de los cambios producidos.

La convergencia lingüística plantea, por lo tanto, la eliminación de rasgos no equivalentes en el contraste entre las variedades en contacto, y puede suponer igualmente la introducción de rasgos y material foráneo que generará una mayor semejanza entre las variedades origen y objeto de los fenómenos. Por ello, el resultado contemplado podrá ser también definido como de ruptura formal por asimilación.

2. Los cambios formales producidos inciden sobre la viabilidad comunicativa de la variedad nativa, en la medida en que ésta se revela como un código funcionalmente limitado en sus finalidades comunicativas internas, y estilísticamente empobrecido por cuanto decrecen o desaparecen las opciones formales previamente en uso.

En este sentido, con la variedad nativa cada vez habrá menos cosas que se puedan decir, y cada vez se podrán decir con menos recursos propios ${ }^{4}$.

Siendo éste el horizonte de trabajo cuando se intentan analizar los efectos del cambio inducido en general, algunos autores han tendido a restringir y a diferenciar su alcance cualitativo. Y aunque prevalece el paradigma simplificador, no todos los fenómenos de cambio inducido e interferencia tienen como resultado evidente la ruptura formal de las estructuras nativas ${ }^{5}$.

Con respecto a ello, sin embargo, hay que convenir en que, con todas las matizaciones que se han realizado, el paradigma de estudio descansa en una interpretación lineal de los efectos de aquellos fenómenos; y, lo que es más importante, se omite cual-

${ }^{2}$ Véanse Dessler 1987, p. 92; 1988, p. 188.

${ }^{3}$ Véanse los trabajos panorámicos de Campbell \& Muntzell 1989 y Hagen \& De Bot 1990 para una tipología tentativa de dichos fenómenos de simplificación y/o regularización en distintas lenguas nativas.

${ }^{4}$ Véanse Dressler 1987; Dressler \& Wodak 1977; Mougeon \& BENIAK 1989.

${ }^{5}$ Véanse Trudgill 1976-1977; Mougeon \& Beniak 1989. 
quier intento de profundización en la "ecología" de tales fenómenos, a los que sólo se les adjudica significación mediante su ubicación en un proceso continuado de selección y decaída ${ }^{6}$.

$\mathrm{Y}$ aun cuando se advierten los matices que arriba hemos señalado con respecto a la ruptura formal, que no es un resultado taxativo, en lo que sí parece producirse un consenso general es en cuanto a la pérdida de eficacia funcional y de diversidad estilística emanada tanto de los cambios formales operados como del propio contexto restrictivo de contacto, siempre desde la perspectiva de la lengua recesiva.

A pesar de esas hipótesis planteadas y de esas líneas de investigación recurrentes, es posible realizar un intento de argumentación que incida en otros parámetros de interpretación teórica. Por una parte, intentar explicar los efectos de los fenómenos de contacto según su hipotética resolución no deja de ser un ejercicio prospectivo con un nivel de predicción poco evidente. Por otro lado, el paradigma simplificador se basa en una interpretación reduccionista del cambio inducido, sometido a un tipo de análisis empírico que no profundiza en los efectos socio-semánticos y pragmáticos implicados en tales fenómenos, siempre en relación con los grupos y categorías de hablantes que actualizan el patrimonio lingüístico nativo.

\section{LA RELATIVIDAD DE LAS DEPREDACIONES FORMALES}

A lo largo de las diversas aportaciones realizadas sobre los efectos del contacto interlingüístico, se ha manejado un conjunto de datos empíricos que muestra los efectos de distintos fenómenos de cambio lingüístico inducido y de diferentes mecanismos de interferencias. Esos datos, incluidos en diferentes investigaciones parciales o en recopilaciones generales, pueden ser aducidos aquí bajo un replanteamiento formal que nos permita relativizar los efectos de tales fenómenos. A partir de estos datos, presentamos cuatro conjuntos de evidencias que tienden a mitigar los presupuestos sobre los que se asientan las interpretaciones basadas en 1 y 2.

${ }^{6}$ En la medida en que, como señalan Dressler \& WodAK 1977, p. 9, los fenómenos de reducción y adaptación lingüística deben ser interpretados como signos de "obsolescencia", al tiempo que aceleran el proceso de extinción. 


\subsection{Cambios inducidos y compensaciones internas}

El principio general del cambio inducido según el cual, y a diferencia del cambio lingüístico espontáneo, aquél no genera compensaciones internas, presenta innumerables excepciones. $\mathrm{O}$ lo que es lo mismo: su validez como esquema regular de los cambios inducidos no parece tener un óptimo alcance descriptivo general. E incluso podríamos establecer una caracterización inversa: un descenso en la complejidad estructural de un paradigma particular es generalmente compensado mediante un incremento formal en el mismo componente afectado en otra área de la gramática nativa ${ }^{7}$.

De hecho, como han señalado algunos autores, es difícil diferenciar el cambio inducido del cambio espontáneo según el criterio de la falta de compensación, ya que tal diferencia cabe plantearla en términos de la cantidad de cambios producidos, y de la rapidez de su difusión (Dorian 1981, pp. 151-152).

Aceptando la diferencia teórica propuesta (Trudgill 1976-1977) entre "simplificación" (pérdida con compensación) y "reducción" (pérdida sin compensación), parece plausible argumentar que este último fenómeno denota una situación de contacto extrema, por cuanto el nivel de desintegración grupal o comunitario nativo que revela s éra en extremo $\mathrm{radical}^{8}$. Pero en situaciones de contacto socialmente menos dramáticas, es de esperar una tendencia mayor hacia el resultado simplificador, entendido en el sentido de pérdidas que generen algún tipo de compensación.

Un buen número de casos que pueden ser mostrados como ejemplos de cambios con compensación se enmarcan dentro de lo que podemos denominar la "transferencia formal"': la expresión de una función gramatical determinada es transferida a otra forma lingüística. $Y$ esta transferencia puede verse realizada en el propio componente simplificado o en otra área estructural nativa.

En arvanítika (Tsitsipis 1983, p. 300), la función desarrollada por el modo optativo, aunque sobrevive en algunas formas fosili-

7 Véase Hagen \& De Bot 1990, pp. 143-144.

${ }^{8}$ Como puede ser tipificado en los contextos de las lenguas pidgin, o como también se indica en buena parte de las situaciones de extinción lingǘstica: "This [reduction] exemplifies a distinctive feature of the language death process, $i$. e. reduction in the structure of a decaying language is not always compensated for by structural expansion and elaborate elsewhere in the system" (Schmidt 1985, p. 229). 
zadas, es realizada por los hablantes menos fluidos mediante formas del modo subjuntivo.

En esta misma variedad recesiva, la sustitución de la construcción con gerundio por otra construcción con conjunción (ibid., pp. 300-301), la expresión de futuro y de imperativo en dyirbal realizada con otros recursos léxicos (Schmidt 1985, p. 229), la sustitución del genitivo por frases preposicionales en el gaélico escocés (Dorian 1981, pp. 130-131), la reducción de alomorfos y la pérdida del caso ergativo, provocando un orden sintáctico más rígido, en dyirbal (Schmidt 1985, p. 229) ${ }^{9}$, todos estos casos son una pequeña muestra que ejemplifica la consecución de soluciones compensatorias fuera del área gramatical donde se producen los cambios lingüísticos simplificadores.

$\mathrm{Y}$ volviendo al ejemplo del modo optativo en arvanítika, ese significado modal también puede ser vehiculado por una construcción perifrástica a partir de un verbo impersonal nativo (Trudgill 1976-1977, p. 118). En este caso, y según este autor, el cambio producido no sólo genera una compensación interna dentro del componente morfológico, sino que el resultado aleanzado provoca la adición de cierta cantidad de complejidad interna dentro del componente sintáctico ${ }^{10}$.

Y para finalizar este breve apunte quizá también podamos plantear como ejemplos de cambios compensados de forma interna ciertos casos de lexicalización y de especialización funcional ${ }^{11}$. El proceso resultante en ambos casos, lejos del colapso formal por la desaparición de la correspondiente forma nativa debilitada, es la especiahzación funcional o la lexicalización del elemento nativo, que de este modo no pierde, aunque sí reduce, su operatividad funcional.

${ }^{9}$ Al igual que en dyirbal, Campbell \& Muntzell 1989, pp. 193-194 documentan un desarrollo similar - desaparición del caso ergativo y consiguiente aumento de la rigidez sintáctica- en finés americano.

${ }^{10}$ Este caso sería un ejemplo de lo que P. TRUdGill 1976-1977 define como "simplificación con coste".

${ }^{11}$ En algunos procesos de lexicalización (MoOre 1988), formas lingüísticas con una relativa complejiad morfosintáctica son reanalizadas como raíces simples preparadas para recibir alomorfos de flexión; en los casos de especialización funcional (SILVA-CoRbalán 1986), una forma nativa se extiende a expensas de otra forma, también nativa, que se debilita, siguiendo la analogía con el esquema correspondiente de la lengua foránea. 


\subsection{Simplificación y complicación estructural}

Otro argumento que podemos aportar se basa en lo que se ha denominado asimetría en los esquemas de cambio producidos. Se ha señalado ${ }^{12}$ que al lado de fenómenos de simplificación morfológica se mantienen áreas de complejidad y que incluso dentro de un mismo paradigma no todos los rasgos y/o elementos se muestran igualmente "debilitados" por el efecto de los cambios inducidos ${ }^{13}$.

A. Schmidt, en sus conclusiones sobre la obsolescencia del dyirbal, indica que un desarrollo asimétrico de esta naturaleza -áreas de simplificación estructural conviviendo con el mantenimiento de paradigmas altamente complejos - es una característica inherente a los esquemas del cambio inducido en aquellos procesos de declive lingüístico. Y como ya pudimos observar también en el punto anterior (cf. 2.1), la compensación de un cambio simplificador puede llegar a provocar un aumento de la complejidad estructural interna (cf. Trudgill 1976-1977).

De hecho, Hill (1989, p. 155) señala que el nivel de reducción en los índices de relativización sintáctica en náhuatl no está acompañado por la simplificación gramatical en sentido estricto, ya que: a) se produce un mayor número de opciones para la posible formación de cláusulas de relativo, y $b$ ) se genera una mayor complejidad sintáctica debido a la extensión de una cláusula es-tructuralmente marcada, en el sentido de menos transparente desde una perspectiva gramatical.

En este tipo de casos la simplificación gramatical conlleva un aumento de la complejidad interna, en la medida en que aumentan las formas lingüísticas esperables y en la medida en que la fórmula gramatical resultante - por ser la más utilizada- representa una opción formal, cuando menos, más opaca.

Por lo tanto, los procesos simplificadores en marcha ni impiden la retención de áreas de complejidad estructural, ni impiden que tales simplificaciones se conviertan posteriormente en una potencial fuente de "complicaciones". Y aunque no siempre puedan ser aportados ejemplos de este tipo, en todo caso parece prevale-

12 Véase Schmidt 1985, pp. 231-232.

13 DORIAN 1983, p. 165, realiza una apreciación similar con respecto a los procesos simplificatorios detectados en los semilingües ("semispeakers"), cuando señala que en muchos casos "they do not simplify as much as they well might". 
cer un esquema asimétrico como el señalado arriba: complicación y simplificación conviven en constante reorganización.

\subsection{Simplificación y transparencia estructural}

Hasta ahora hemos considerado los resultados del cambio inducido haciendo abstracción de los contextos socio-comunitario y lingüístico en donde se producían. Sin embargo, si aquellos resultados - simplificaciones, regularizaciones, pérdidas, etc.son entendidos como ejemplos de una tendencia a reducir la complejidad estructural existente - por cuanto ése es el resultado final-, tendremos que convenir que en muchos casos los fenómenos de reducción formal arrancan de una realidad obvia: estructuras definidas como "complejas" tienden a ser reinterpretadas en aras de una mayor univocidad forma-significado.

Tomando con precaución la definición de "transparencia estructural", y recogiendo la perspectiva adoptada por Hill (1978) sobre la necesidad de tener en cuenta las dinámicas socioculturales de los hablantes que actualizan cambios "transparentes", la búsqueda de un mayor grado de univocidad entre la forma y la función gramatical no parte de una realidad lingüística objetivamente compleja, sino que más bien se relaciona con la capacidad de los hablantes para poder adecuar una forma lingüística concreta con un contexto de uso cada vez más limitado y cada vez también más condicionado por la presión lingüística foránea.

Cuando la lengua minoritaria se emplea en espacios cada vez más reducidos, el desfase entre la forma lingüística y su uso social provoca un mayor potencial de reinterpretación interna, en aras de conjugar aquellos dos parámetros formales y funcionales. Y será aquí donde el concepto de transparencia, aplicado a esta parcela particular, comenzará a tener plena operatividad.

Dentro de un contexto de esta naturaleza, en el cual uso y forma lingüística nativa se hallan abiertamente disociados, podremos argumentar que los cambios inducidos se articularán mediante rasgos como "opacidad" y "transparencia". Si, como señala Silva-Corbalán (1986, p. 600), las reglas que denotan un aspecto gramatical particular devienen funcionalmente opacas, estaremos ante una potencial fuente de reinterpretación formal. Y en este sentido la transparencia estructural se podrá enunciar como un proceso tendente a simplificar determinadas estructuras 
gramaticales $^{14}$, que de esta manera serán aprehendidas con mayor facilidad en términos de aprendizaje y uso, una vez la ambigüedad inherente haya quedado desactivada mediante los consiguientes cambios internos ${ }^{15}$.

Un buen ejemplo de este tipo de procesos nos lo ofrece Dorian (1985) con respecto al imperativo y al vocativo en el dialecto gaéhco-escocés estudiado. En ambos ejemplos, el sistema de marcas de caso y de flexión, respectivamente, presentan una alta variabilidad condicionada principalmente por el contexto fonético de aparición, según el cual es necesario seleccionar un determinado alomorfo flexivo nominal - en el vocativo- o verbal -en el imperativo negativo y positivo.

De este proceso de reducción alomórfica, podemos subrayar tres cuestiones de interés: 1) el proceso simplificador reseñado está más cerca de la regularización formal que de la pérdida funcional; 2) mediante este proceso regularizador, el vocativo y los dos paradigmas del modo imperativo alcanzan una mayor uniformidad en consonancia con el resto del sistema paradigmático en donde se insertan ${ }^{16}$, con una ligera pérdida de riqueza sintática pero resultando así una mayor regularización general en la selección de variantes; 3) el resultado general de los cambios producidos presenta, según la autora, una pérdida poco reseñable de opciones formales y, lo que es más importante, ninguna categoría gramatical se ha visto limitada en su capacidad "comunicativa" o "informativa".

Aunque quizá sí sea posible rastrear tales pérdidas, éstas afectan a oposiciones que presentan un bajo índice de rentabilidad funcional, ya que afectan a paradigmas concretos altamente idiosmcráticos y/o a paradigmas con un alto grado de redundancia. Y siendo así, el resultado alcanzado mediante los cambios producidos implica un mayor grado de regularización que, unido a una pérdida poco importante de opciones en uso, redunda en una mayor transparencia estructural y en un mayor nivel de ac-

14 Aquellas que puedan ser definidas como "áreas de innecesaria - no funcional-complicación"' (ScHMidt 1985, p. 230).

15 Véase en esta misma línea MAANDl 1989, p. 230.

16 Con respecto al imperativo "positivo", " although ESG "loses" a verbal number distinction, it "gains" a verbal system in which number marking is consistent in its absence" (DORIAN 1985, p. 170); y con respecto al imperativo negativo, "all verbs can be negated on exactly the same pattern" (ibid., pp. 170-171). 
cesibilidad para los hablantes, dadas las condiciones en las que se desenvuelve su actividad lingüística.

A pesar de lo dicho aquí, convendrá tener en cuenta dos cuestiones. En primer lugar, habrá que observar la cantidad y extensión de tales cambios regularizadores: la eficacia comunicativa podría verse seriamente afectada si el proceso simplificador afectase a amplios espacios estructurales o si produjese deficiencias insoslayables en los medios de expresión nativos. En segundo lugar, este tipo de regularizaciones, aunque muy extendidas en los contextos de contacto, no son comparables con las que acontecen en situaciones como las reseñadas en las lenguas pidgin, como ya ha sido señalado por diversos autores.

\subsection{Cambios inducidos y adiciones estructurales}

Como hemos reseñado arriba, los cambios simplificadores detectados en una variedad recesiva suponen un proceso de recesión de rasgos y/o formas nativas que cesan en su operatividad estructural. En la raíz de este proceso, sin embargo, puede estar situado como inicio un fenómeno de interferencia foránea, de tal manera que una forma nativa (oposición, rasgo, paradigma, etc.) sucumbe ante la competición con otra forma más o menos equivalente pero de origen foráneo.

En este tipo de situaciones formales, ampliamente conocidas entre los que han trabajado sobre el paradigma de la interferencia, el proceso simplificador arranca de un esquema de convivencia sincrónica entre material formal y/o funcionalmente equivalente en la variedad objeto y la variedad fuente de las interferencias, al menos en determinados estados sincrónicos. Tal convivencia ha sido enunciada, siguiendo el patrón simplificador, en términos de "provisionalidad", en la medida en que las adiciones foráneas serán el resultado único y taxativo a medio o corto plazo ${ }^{17}$.

Por ello, el esquema de simplificación formal no sólo se articula mediante pérdidas generalizadas, sino que, en este tipo de casos, es necesario hablar de adiciones efectivas de material, aunque ese contexto sea enunciado en términos de provisionalidad diacrónica.

En cuanto al resultado de este conjunto de adiciones foráneas -bien sean “actos de recepción”' (Campbell \& Muntzell 1989,

${ }_{17}$ Como sugiere Denison 1977, pp. 19-20. 
pp. 193-194; Campbell 1987, p. 253), bien se trate de "transferencias estructurales"' (Hagen \& De Bot 1990, pp. 143 ss.)-, siempre se ha tendido a realizar una interpretación en términos de corrupción formal, en la medida en que las formas nativas son sustituidas por las foráneas, y por cuanto tales cambios, así consolidados, redundan en hacer de la lengua nativa un especimen estructuralmente más próximo a la variedad fuente de las adiciones; en otros casos, sin embargo, los fenómenos de interferencia serán enunciados en términos de pérdida "menor", como en el caso de especialización funcional ${ }^{18}$.

\section{Cambios lingüísticos Inducidos en el Contacto Gallego-Castellano}

El contacto entre el gallego y el castellano de Galicia, prolongado en el tiempo e intenso para la mayor parte de comunidades y grupos gallegohablantes actuales, nos permite recoger diferentes ejemplos de fenómenos lingüísticos en gallego - la variedad nativa - que documentan la pujanza de la presión del castellano - la variedad foránea en el contacto.

3.1. Para la presente exposición, nos centraremos en dos fenómenos de cambio inducido que afectan al componente fonético del gallego. En primer lugar, el gallego cuenta dentro de su sistema vocálico ${ }^{19}$ con las formas $\left.[\epsilon],[]\right]$ - vocal anterior abierta y vocal posterior abierta respectivamente-, y con los diptongos crecientes [je], [we]. Ambos pares de variantes $-[\epsilon]$ y [je] por un lado, [3] y [we] por otro- se ubican en contextos léxicos de aparición sincrónica y diacrónicamente determinados, de manera que la aparición de la variante vocálica o de la correspondiente forma diptongada está establecida de una manera taxativa.

En la descripción estructural del gallego, $[\epsilon]$ y $[j e]$ aparecen en contextos léxicos mutuamente excluyentes, como podemos ver en los ejemplos de (1):

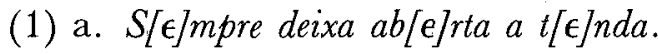

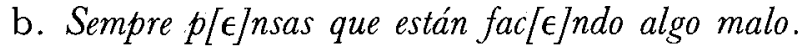

c. Ponte qu[je]to e se obed[je]nte.

18 Véase SILVA-Cor balán 1986.

19 Véase Álvarez et al. 1986. 
En los elementos léxicos, la selección de una u otra variante depende del rasgo $+/-$ culto correspondiente a la base léxica. Ello permite asignar la variante vocálica $[\epsilon]$ para los elementos /-culto/ -como en (1)a-, y [je] para las bases léxicas /+culto/ - como en (1)c. En la flexión verbal, todo verbo que contenga una vocal -E- en la raíz verbal o como vocal temática, mantiene invariable e inalteradamente su manifestación como $[\epsilon]$ en toda la conjugación - como ocurre en (1)b.

De igual forma, la aparición de [ग] y [we] está condicionada por parámetros semejantes - rasgo $+/$ - culto de la base léxica y manifestación de -O- como [3] en toda la conjugación verbal-, como podemos ver en (2):

(2) a. A p/J/rta tiña o pomo s/J/lto.

b. Non p[ว]des facer que c[j]za máis rápido.

c. $O$ sec[we]stro deixoulle graves consec[we]ncias.

Comparando sus frecuencias de aparición en el gallego actual, las variantes vocálicas registran una mayor presencia que los diptongos, los cuales poseen unos contextos más restringidos de aparición, consecuencia directa de la resistencia estructural del gallego a la pronunciación de los diptongos crecientes ${ }^{20}$.

El cambio detectado, que denominamos regla de diptongación, implica la introducción de los diptongos [je], [we] en aquellos contextos, como los ejemplificados en (1)a, (1)b, (2)a y (2)b, en los que el gallego prevé la única selección posible de las formas vocálicas $[\epsilon],[\jmath]$. Este proceso de diptongación se corresponde con lo que ocurre en castellano: la introducción de [je] y [we] en los coritextos propios de [ $\epsilon]$ y [2] sigue la distribución existente en aquella lengua.

En segundo lugar, dentro del sistema consonántico, el gallego presenta una variante fricativa palatal sorda [\}] en aquellos contextos fonéticos delimitados por la evolución diacrónica.

(3) a. Dei[J]ei o [〕Jamón enriba da mesa.

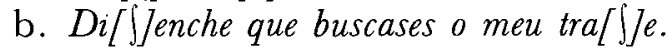

La variante fricativa velar sorda $[\mathrm{x}]$ no existe en gallego más que como una posible realización de un rasgo dialectal gallego

${ }^{20}$ Cf. ibid., p. 39. 
conocido como gheada ${ }^{21}$. Aunque la variante velar no es completamente desconocida en gallego, ya que se registra como rasgo de estratificación dialectal, en todo caso no se halla en distribución complementaria con la variante palatal []$^{22}$.

El cambio producido, que denominamos regla de velarización, implica la introducción de la variante fricativa velar sorda $[\mathrm{x}]$ en todos aquellos contextos en los que el gallego prevé, como única forma posible, la presencia de la variante fricativa palatal sorda [ [].

La aplicación de la regla de velarización se produce en aquellos contextos léxicos en los que también se da una correspondencia interlingüística entre gallego y castellano, como ocurre en los ejemplos presentados en (3) $3{ }^{23}$.

Tanto con respecto a la regla de diptongación como a la de velarización, estamos ante ejemplos de cambios inducidos de naturaleza fonética. Hay que convenir, sin embargo, en que el origen de ambos fenómenos debió estar situado en el componente léxico del gallego - mediante la introducción de aquellas variantes a través de préstamos procedentes del castellano. A partir de ese origen puramente léxico, la introducción de las variantes foráneas contenidas en segmentos del castellano se generaliza a todos los contextos posibles de ocurrencia, como son buena prueba la presencia de [je], [we] y [x] en adjetivos, adverbios y verbos del gallego (además, por supuesto, de en la categoría nominal).

Por ello, lo que en un principio podía ser analizado como simples interferencias léxicas, puede ser ya considerado, por lo menos en agrupaciones sociales con un alto nivel de contacto interlingüístico, como un ejemplo de variación fonética interna al repertorio de los hablantes.

En ambas reglas, estamos ante una reorganización interna

${ }^{21}$ La gheada consiste en la aspiración de /g/ en todos los contextos fonéticos de aparición; la realización más extendida de la gheada es un sonido aspirado laríngeo $/ h /$, pero también se recogen variantes de la gheada de carácter velar, próximas a la $-j$ - del castellano.

22 En el paso del latín a las dos lenguas romances, mientras que el gallego mantuvo aquella variante palatal, el castellano evolucionó hacia la variante velar. En la sincronía actual, gallego y castellano poseen dos realizaciones consonánticas diferentes para los mismos contextos fonéticos de aparición que sean interlingüísticamente equivalentes.

${ }^{23}$ Por ello, quedan fuera de la regla de velarización propuesta aquellos contextos de aparición de / / q que no impliquen la correspondencia con $/ \mathrm{x} /$ en castellano. 
del gallego producida a instancias de la distribución planteada en castellano: la introducción de los diptongos [je], [we] y de la variante $[\mathrm{x}]$ en contextos no previstos por las reglas del gallego sigue la distribución existente en castellano. Y mientras que la regla de diptongación supone la alteración de la distribución complementaria de las variantes, en la regla de velarización asistimos a la introducción de una forma fonética que, excepto como rasgo dialectal y con otra distribución, no existe en la descripción fonética del gallego.

3.2. El grupo de hablantes en los que se ha observado el funcionamiento de ambas reglas puede ser definido como una agrupación social relativamente homogénea en proceso de mantenimiento de la variedad nativa. Esto puede ser argumentado teniendo en cuenta que se trata de hablantes de una Alta Edad Media compartida, que pertenecen a una generación que fue objeto de la transmisión primaria del gallego ${ }^{24}$, y que es esa lengua la que hoy sustenta la propia configuración intragrupal.

Ello no impide, sin embargo, que estos hablantes posean, en diferente grado, un amplio conocimiento del castellano, y que lleguen a utilizarlo con diferente intensidad bajo determinadas condiciones de interacción ${ }^{25}$. A pesar de ello, es plausible argumentar que esta agrupación observada presenta unas pautas de actuación sociolingüística ubicadas alrededor del concepto de mantenimiento social de la variedad nativa, por cuanto el gallego vehicula en exclusiva los intercambios intragrupales y esa constancia constituye una característica inherente a la propia configuración nuclear de la comunidad estudiada.

Analizando la variación producida en las reglas de diptongación y de velarización ${ }^{26}$, podemos apuntar un mayor predominio de las variantes nativas en verbos, adjetivos y adverbios, mientras que en los elementos nominales la variación apuntada presenta una mayor proximidad entre las frecuencias absolutas de las dos variantes posibles en cada regla.

24 Véase Argente \& Lorenzo $1991 \mathrm{~b}$.

${ }^{25}$ Cf. Argente \& Lorenzo 1991a.

${ }^{26}$ Para datos sobre el corpus, número y tipo de hablantes, así como los contextos de recogida del material lingüístico, véase LoRENzo 1990. 
TABLA 1

Frecuencia de las variantes

\begin{tabular}{|c|c|c|c|c|c|c|c|c|}
\hline & \multicolumn{4}{|c|}{ Regla de diptongación } & \multicolumn{4}{|c|}{ Regla de velarización } \\
\hline & \multicolumn{2}{|c|}{$[\epsilon, \supset]$} & \multicolumn{2}{|c|}{$[\mathrm{je}, \mathrm{we}]$} & \multicolumn{2}{|c|}{ [.] } & \multicolumn{2}{|c|}{$[x]$} \\
\hline & $\%$ & $\mathrm{n}=$ & $\%$ & $\mathrm{n}=$ & $\%$ & $\mathrm{n}=$ & $\%$ & $\mathrm{n}=$ \\
\hline $\mathrm{N}$ & 0.55 & 145 & 0.45 & 119 & 0.44 & 75 & 0.56 & 99 \\
\hline V & 0.90 & 124 & 0.10 & 12 & 0.66 & 41 & 0.34 & 21 \\
\hline \multirow[t]{2}{*}{ A } & 0.83 & 75 & 0.17 & 15 & 0.81 & 35 & 0.19 & 8 \\
\hline & 0.70 & 344 & 0.30 & 146 & 0.54 & 151 & 0.46 & 128 \\
\hline
\end{tabular}

En la tabla 1 podemos observar el amplio predominio de las variantes nativas [j] y $[\epsilon, \partial]$ en verbos, adjetivos y adverbios; en esos contextos, las reglas de diptongación y velarización operan con un bajo rendimiento. En cambio, en los elementos nominales de la regla de diptongación, el porcentaje de las variantes nativas supera por escaso margen la frecuencia de las variantes foráneas -0.55 frente a $0.45-$, mientras que en la regla de velarización la variante $[\mathrm{x}]$ alcanza mayor frecuencia que la forma nativa $\left[\int\right]-0.56$ frente a 0.44 .

Indagando esta tendencia presente en las bases léxicas nominales, la aparición de una u otra variante dentro de cada regla, lejos de ser aleatoria, parece responder a una estratificación basada en los aspectos referenciales contenidos en la pieza léxica en la que se insertan aquellas variantes fonéticas. Estas "esferas referenciales" definen el conjunto de campos semánticos de carácter nominal $-o$ relacionados con referentes nominales-, que referencian actividades, objetos, conceptos, cosas, etc., de la realidad psicosocial del individuo.

Aplicando esta distinción a los elementos nominales, discriminamos dos ámbitos distintivos y diferenciados de referencia semántica: la esfera local y la esfera no local ${ }^{27}$. Esta categorización permite captar la tendencia detectada en la asignación de las variantes fonéticas de cada regla: las variantes [ $\epsilon]$, [j] y [j] predominan en las esferas referenciales "locales", mientras que las variantes [je], [we] y $[\mathrm{x}$ ] predominan en las esferas "no locales".

Así lo podemos comprobar, en primer lugar, dentro de la regla de diptongación:

${ }^{27}$ La esfera local designaría el conjunto de campos semánticos y de elementos lingǘsticos que remiten al entorno local del hablante. La esfera "no local" remitiría al universo semántico no inmediato para el individuo, con respecto al cual se tiene (o se quiere) una determinada distancia simbólica. 


$$
\text { TABLA } 2
$$

Frecuencia de las variantes en elementos nominales Regla de diptongación

\begin{tabular}{rrrrr}
\hline & \multicolumn{2}{c}{$[\epsilon$, j] } & \multicolumn{2}{c}{$[$ [je, we] } \\
& $\%$ & $\mathrm{n}=$ & $\%$ & $\mathrm{n}=$ \\
\hline + Local & 0.73 & 111 & 0.27 & 41 \\
- Local & 0.30 & 34 & 0.70 & 78 \\
& 0.55 & 145 & 0.45 & 119 \\
\hline
\end{tabular}

La referencia semántica contenida en la pieza léxica correspondiente remite a realidades diferenciadas, y de esta forma predispone la aparición de una u otra variante en la regla de diptongación, como podemos comprobar en los siguientes ejemplos extraidos del corpas:

(4) Contextos $(+$ Local $)$

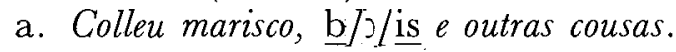

b. Baixábamos as vigas de $\mathrm{f} / \epsilon /$ rro para facer cortaf/J/gos.

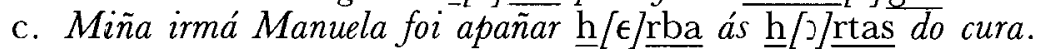

d. Antes habia moitos $\underline{\mathrm{h}}[\mathrm{J}] \underline{\text { is }}$ labrando... faciamos moitas cousas $n a \underline{\mathrm{t}}[\epsilon] \underline{\mathrm{rra}}$, veña á $\underline{\mathrm{h}}[\epsilon] \underline{\mathrm{rba}}$, veña ó monte...

(5). Contextos (-Local)

a. Inda tuven que pagar trinta $e$ seis mil pesetas a has $[j \in]$ nda.

b. Sempre estábamos acostumbrados a dous $\underline{\mathrm{s}}[\mathrm{we}] \underline{\mathrm{l} \text { dos. }}$.

c. Sacáronme o tratam $/ j e /$ nto porque decían que xa estaba ben.

Una distribución de la misma naturaleza podemos analizarla dentro de la regla de velarización:

TABLA 3

Frecuencia de las variantes en elementos nominales Regla de velarización

\begin{tabular}{ccccc}
\hline & \multicolumn{2}{c}{$\%^{\prime / \prime}$} & \multicolumn{2}{c}{$/ \mathrm{x} /$} \\
& & $\mathrm{n}=$ & $\%$ & $\mathrm{n}=$ \\
\hline + Local & 0.64 & 52 & 0.36 & 29 \\
- Local & 0.25 & 23 & 0.75 & 70 \\
& 0.43 & 75 & 0.57 & 99 \\
\hline
\end{tabular}


También aquí el rasgo + / - local asociado a la base léxica nominal alienta la estratificación entre las dos variantes de la regla de velarización, dándose una mayor frecuencia de aplicación de la regla en la esfera (-local), mientras que en la esfera referencial ( + local) su operatividad decrece considerablemente - ya que [J] alcanza una frecuencia de 0.64.

En los ejemplos siguientes podemos comprobar la ubicación semánticamente determinada de las variantes dentro de la regla de velarización:

(6) Contextos $(+$ Local $)$

a. (Eu) vivo na bai $[$ J Jada do Espiritu Santo.

b. Cando plantou fogo na gran/ /Ja moi poucos nos axudaron.

c. A miña nai tuvo que ir ó l//ornal, a fase de todo.

d. Cando eu era nova tamén anduven ó me $\iint j \underline{\text { lión. }}$

(7) Contextos (-Local)
a. Fun a Vigo a ur $[x]$ encias, en ur $[x]$ encias non me quixeron atender.
b. Eu son mui reli/x/ioso. A reli/x $x$ ión sempre foi en castellano, claro, eu rezo en castellano.
c. Agora estou pre $/ x /$ ubilado, a punto de [x/ubilarme.
d. Antes non habia ese libertina $[x]$ e que hai agora.

Las frecuencias mostradas en las tablas 1-3 ofrecen una regularidad considerable: las variantes nativas predominan en los elementos no-nominales, mientras que en la categoría nominal las variantes se hallan estratificadas según el referente socio-semántico asociado.

Sin embargo, y a pesar de esta regularidad manifiesta, no podemos obviar la incipiente fluctuación que arrojan estos datos. En verbos, adjetivos y adverbios, como podemos ver en la tabla 1, las variantes foráneas llegan a alcanzar una frecuencia relativa máxima de 0.34 (en la variante $[\mathrm{x}$ ] de los elementos verbales). Igualmente, en las bases nominales, las variantes [je], [we] y [x] penetran en las esferas ( + local), mientras que las variantes $[\epsilon]$, [ว] y [j] se rastrean en los contextos (-local). Esta fluctuación puede ser indicativa de una reorganización en marcha o de un cambio que aún no se ha completado. El resultado final de este 
proceso, sin embargo, no parece tener una dirección evidente, en la medida en que la variación producida afecta a ambos contextos referenciales por igual.

Pero además de esta estratificación de tipo socio-semántico, en el corpus recogido podemos analizar ejemplos de especializaciones semánticas ${ }^{28}$ con diverso contenido: dobletes léxicos que articulan un diferente significado según el empleo de una u otra variante dentro de cada regla, como podemos comprobar brevemente con algunos ejemplos a continuación:

(8) "escola-escuela"

a. Cando salín da esc [J]la das monxas fixen os estudios normales.

b. Entonces eu vin aquí, á esc [we]la... Entonces viñamos á esc/wejla, que habia moitos rapaces aquí, e tuvemos unha maestra moi boa.

c. Estuven na esc/J/la de aí arriba. Era unha esciwejla así, de pago.

(9) "fogo-fuego(s)"

a. Eu xente que veña a apagar o $\mathrm{f} / \mathrm{J}$ go vouche decir quen'foi: ...

b. ¿Que tedes o $\mathrm{f} / \mathrm{J} / \mathrm{go}$ diante da casa e estades durmindo!', decíannos.

c. Fáltalle un dedo de ir a buscar os $\underline{\mathrm{f}}[\mathrm{w} w e] \mathrm{gos}$.

En los ejemplos de (8), esc[we]la vehicula dos posibles acepciones: 1) lugar del entorno local donde se recibe educación; 2) ámbito institucionalizado de educación. En el ejemplo (8)b podemos comprobar estas dos acepciones mediante el uso de la variante [we]. Por su parte, esc[2] la sólo es empleada con la primera acepción, como podemos comprobar en (8)a. Finalmente, en (8)c observamos el empleo sucesivo de la misma pieza léxica con ambas variantes: [0] con la primera acepción, y [we] con la segunda.

En (9), la distinción "fogo/fuego(s)" radica igualmente en el diferente objeto al que remite. Entre fogo "algo que arde" y fuego " artificial, propio de las fiestas", queda establecida una distin-

${ }^{28}$ Por especialización semántica significamos aquellos dobletes léxicos funcionalmente especializados que son empleados con diferente significado situacional según la variante fonética utilizada. 
ción referencial para la cual, al igual que en (8), la variante fonética empleada sirve de delimitador contextual.

Pero el empleo de las variantes foráneas y nativas no sólo permite vehicular diferentes significados semánticamente motivados, sino que también es posible aducir ejemplos de variación pragmáticamente condicionada. En estos casos, y siempre en relación con fenómenos de énfasis ${ }^{29}$, el empleo de las variantes de cada regla conlleva una marca contextual de significados interactivamente relevantes en los intercambios efectuados, como podemos analizar en los siguientes fragmentos:

(10) Tu fighate, que non ghastan un duro nos barcos, $e(a)$ nós en tódolos via/b]es teñen que reparar(nos) o barco. $E$ neste último via $[x]$ e ainda estuvemos tres dias parados.

(11) Estabamos dous homes solos, coa máquina que arrea vinteseis peixes por minuto, e aquí.estou. . ¿ ent $[\epsilon]$ ndes? . . E si lle contestas algo $x a$ che están dando o pase, xa te amenazan cun parche, ¿ent $[j e]$ ndes? ...e non podes ir a ningún lado, ¿ent $[j e]$ ndes?

(12) (Locut. 1): . . porque os médicos saben muito, saben o que ti queres tomar e cho dan.

(Locut. 2): Iso chámase analítica psicoló/[j] ica.

(Locut. 1): Si, eso, analítica psicoló $/ x /$ ica.

En estos tres ejemplos, los hablantes alternan el uso de una misma pieza léxica con las dos variantes de cada regla. $\mathrm{Y}$ este empleo enfático se produce mediante el contraste sintagmático entre ambos elementos sucesivos y mediante su ubicación secuencial: el elemento enfatizador está situado a continuación del elemento de contraste. Y es interesante observar cómo el segmento enfatizador siempre contiene una variante "no nativa" - [je] en (11); [x] en (10) y (12).

Lo relevante en estos casos de énfasis no sólo lo constituye el uso alternante de las variantes en la búsqueda de una significación contextual, sino igualmente el hecho de que ese contraste sea sucesivo dentro del discurso producido, y que el mecanismo de énfasis sea activado mediante el empleo de las variantes "no nativas" de ambas reglas.

${ }^{29}$ Por énfasis entendemos el proceso de focalización contextual de una pieza léxica dentro de la cadena discursiva. 
3.3. ¿Qué hipótesis, a partir de los fenómenos analizados en el subapartado anterior, podemos inferir sobre los efectos funcionales y formales del contacto?

En primer lugar, la alternancia entre variantes dentro de cada regla define la norma lingüística local operativa en el seno de la agrupación social analizada. Esta norma local, articulada alrededor del empleo alternante de formas nativas y formas "foráneas" (en lo relativo a su distribución efectiva), se aleja de los patrones fonéticos descritos para el gallego. Y aún más: alejándose de ese esquema teórico se aproxima a la norma presente en castellano.

Este doble proceso simultáneo - configuración de una norma local operativa y aproximación de ésta a aquélla presente en la otra variedad en contacto - puede ser indicativo de una dinámica de convergencia interlingüística en marcha en cada una de las reglas consideradas, en la medida en que los cambios operados siguen la dirección presente en castellano.

En segundo lugar, esta hipotética conjunción interlingüística permite la realización de un conjunto de funciones socio-semánticas y pramáticas de cierta relevancia para los hablantes implicados, que tienden a reorganizar las reglas de partida con el fin de adecuarlas a sus necesidades de comunicación diaria. Es decir, mediante la introducción de [je], [we] y [x] en alternancia con las

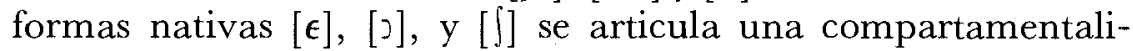
zación comunitariamente compartida de valores asociados con aquellas formas, bien sea por medio de rasgos referenciales, bien sea por medio de contrastes contextualizados - como se produce en los ejemplos de énfasis.

En tercer lugar, centrándonos en la perspectiva estructural, parece difícil argumentar que tales fenómenos, analizados en el seno de una comunidad de habla en proceso de mantenimiento, supongan un indicio de ruptura formal, al menos si realizamos una consideración sincrónica "no finalista" del proceso. El predominio de las variantes nativas en verbos, adjetivos y adverbios y su ubicación predominante en los elementos nominales según el rasgo (+ local) sugieren una convivencia inestable, pero sociolingüísticamente delimitada. E incluso dentro de la categoría nominal, la fluctuación detectada afecta a ambos conjuntos de

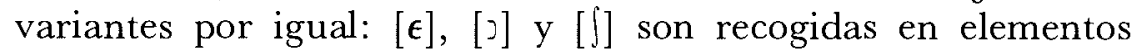
marcados con el rasgo (-local), y al revés: [jel, [we] y [x] penetran en los contextos descritos como ( + local).

Por lo tanto, la dirección del cambio en curso resulta de difícil 
dilucidación, de manera que un análisis basado en la transitoriedad de la distribución presentada por las reglas de diptongación y velarización no parece obtener unos resultados muy evidentes.

En cuarto lugar, lo que aun resulta más difícil de argumentar es que los fenómenos producidos supongan un empobrecimiento funcional, al menos si nos atenemos a la casuística apuntada por la alternancia entre variantes en determinados contextos y con diversos significados referenciales y pragmáticamente motivados. Según lo visto arriba, la alternancia entre variantes - una vez las formas foráneas se integran en la norma local-, ni limita la capacidad referencial del gallego ni imoide la vehiculación de mensajes funcionalmente significativos.

El esquema subyacente a los cambios producidos se orienta hacia la reestructuración de los paradigmas afectados por la disimilitud entre las lenguas en contacto. Pero el efecto producido avanza más hacia la reorganización formal del repertorio - adecuando las reglas lingüísticas nativas a los patrones locales de uso-, que hacia el colpaso formal.

Los cambios en marcha reflejan una situación sincrónica en la cual el alto uso del gallego se realiza en un contexto de fuerte contacto. En este sentido, la funcionalidad que dispensa la variedad recesiva tiene su contrapartida en los cambios formales que se producen.

Por ello, en una situación de mantenimiento los cambios inducidos se generan como adaptaciones formales tendentes a adecuar el contexto sociocultural del contacto con la funcionalidad que se le otorga a la variedad nativa. De esta forma, determinadas agrupaciones sociolingüísticas y determinados fenómenos lingüísticos pueden ser analizados, a modo de hipótesis de trabajo, según un doble eje de interés:

a) los cambios inducidos por el contacto suponen una auténtica reorganización formal de los recursos nativos ante una situación de contacto que garantiza la funcionalidad de la variedad recesiva, pero no así su impermeabilidad formal;

b) mediante ese conjunto de ajustes formales, los hablantes nativos no sólo pueden afrontar con garantías las necesidades socio-comunicativas requeridas, sino que también articulan un conjunto de referencias contextualizadas y significados interactivamente relevantes para sus intercambios verbales.

La reorganización producida - y sus resultados concretosvariarán dependiendo de los grupos sociolingüísticos considerados. En concreto, la agrupación analizada aquí, sometida a un 
proceso de mantenimiento de la variedad vernácula, orienta su norma local hacia una configuración que actualiza la realidad del contacto: es decir, conjugando el alto nivel de uso intragrupal del gallego con la presión lingüística del castellano, la norma local operativa presenta una reorganización estructural tendente a adecuar convenientemente ambos parámetros sociolingüísticos.

\section{Consideraciones finales}

Las hipótesis sobre los resultados del cambio lingüístico inducido basadas en sus efectos estructuralmente rupturistas y estilísticamente empobrecedores no parecen alcanzar un nivel descriptivo excesivamente amplio. Lo que aquí hemos intentado es plantear las dificultades que entraña una descripción de los resultados lingüísticos del contacto ceñida exclusivamente a una interpretación unívoca en forma de pérdida formal y de ineficacia funcional.

En primer lugar, hemos considerado diferentes argumentos que cuestionan la interdependencia entre cambio inducido y ruptura formal. Los cambios inducidos pueden ser compensados con otros desarrollos internos en la variedad objeto de dichos fenómenos. Igualmente, pueden mantenerse niveles altos de simplificación estructural conviviendo con áreas de complejidad contiguas -e incluso la simplificación inicial puede generar, a nivel global, un aumento de la complicación estructural nativa. Del mismo modo, el efecto simplificador detectado puede ser el resultado de la simplificación de estructuras en exceso complejas, debido al desfase planteado entre la forma lingüística nativa y el uso social realizado, en tanto la necesidad de emplear la variedad recesiva puede ser muy superior a las posibilidades de aprehenderla y practicarla de forma normal. Finalmente, no todos los resultados del cambio inducido son simplificatorios, ya que el contacto interlingüístico puede generar adiciones materiales y/o estructurales de difícil catalogación como simplificaciones o reducciones.

En segundo lugar, hemos planteado la ecología de los cambios producidos dentro de un contexto particular de contacto. Considerando los fenómenos de interferencia detectados, y su repercusión sobre el paradigma en que se insertan, hemos mostrado cómo se integran esos fenómenos en el repertorio lingüístico de los hablantes, cuál es la funcionalidad que manifiestan, y cómo se reorganiza el capital lingüístico de los individuos que perseveran en la actividad oral nativa. 
De cara a futuras investigaciones, será de interés considerar el contenido y la dirección de las reorganizaciones estructurales en marcha, observar qué tipo de recursos nativos -y en qué medida - son afectados por el contacto, y cuál es la relación establecida entre aquellos fenómenos lingüísticos derivados del contacto y los procesos sociales de mantenimiento y sustitución lingüística.

Joan A. Argente

Universitat Autònoma de Barcelona

Anxo M. Lorenzo

Universidad de Vigo

\section{BIBLIOGRAFÍA}

Álvarez, R. et al. 1986. Gramática Galega. Galaxia, Vigo.

Argente, J., y A. Lorenzo 1991a. "A relevancia social da alternancia lingüística”, Cadernos de Lingua, 3, 91-109.

— 1991b. "Transmisión lingüística primaria e extensión social dunha lingua minoritaria", 'Actas do I Seminario Internacional sobre Planificación Lingüística. Consello da Cultura Galega, Santiago.

- y Ll. Payrató 1991. "Towards a pragmatic approach to the study of language contact”', Pragmatics, 1, 465-480.

Gampbell, L. 1987. "Syntantic change in Pipil", IJAL, 53, 253-280.

- y M. Muntzell 1989. "The structural consequences of language death", en N. DORIAn 1989.

Denison, N. 1977. "Language death or language suicide?", International Journal of Sociology of Language, 12, 13-22.

Dorian, N. 1981. Language death. University of Pennsylvania Press, Philadelphia

1983. "Natural second language acquisition from the perspective of the study of language death", en R. W. Andersen (ed.), Pidginization and creolization as language acquisition. Newbury House, Rowley, pp. 158-167.

- 1985. "Vocative and imperative in decline", en V. Pieper \& G. Stickel (eds.), Studia linguistica diachronica et synchronica. Mouton, Berlin.

- (ed.) 1989. Investigating language obsolescence. Cambridge University Press, Cambridge.

Dressler, W. 1987. "La mort de les llengües", Limits, 3, 88-97.

- 1988. "Language death", en F. Newmeyer (ed.), Linguistics: The Cambridge survey. T. 4: Language: The socio-cultural context. Cambridge University Press, Cambridge, pp. 184-192.

- y R. WODAK 1977. "Language preservation and language death in Brittany", International Journal of Sociology of Language, 12, 33-45. 
Hagen, A., \& K. Debot 1990. "Structural loss and levelling in minority languages and dialects", Sociolinguistica, 4, 136-149.

HiLl, J. 1978. "Language death, language contact, and language evolution", en W. McCormack \& S. Wurm (eds.), Approaches to language. Anthropological issues. Mouton, The Hague.

- 1989. "The social functions of relativization in obsolescent and nonobsolescent languages", en N. DORIAN 1989.

LoRENzo, A. 1990. O mantemento lingüistico: proceso social e resultados estructurais no contacto galego-castelán. Universitat Autònoma de Barcelona, Barcelona (inédito).

MAANDI, K. 1989. "Estonian among Immigrants in Sweden”, en N. DoRIAN 1989.

Moore, R. 1988. "Lexicalization versus lexical loss in Wasco-Wishram language obsolescence", IJAL, 54, 453-468.

Mougeon, R., \& E. Beniak 1989. "Language contraction and linguistic change: The case of Welland French", en N. DoR IAN 1989.

- 1991. Linguistic consequences of language contact and restriction. Oxford University Press, Oxford.

Schmidt, A. 1985. Young people's Dyirbal. Cambridge University Press, Cambridge.

Silva-Gorbalán, C. 1986. "Bilingualism and language contact: The extension of ESTAR in Los Angeles Spanish", Lan, 62, 587-608.

Thomason, S., \& T. KaUfman 1988. Language contact, creolization and genetic linguistics. University of California Press, Berkeley.

Trudgill, P. 1976-1977. "Creolisation in reverse: Reduction and simplification in the Albanian dialects of Greece", Transactions of the Philological Society, 1976-1977, pp. 32-50; reeditado en On dialect. Social and geographical perspectives, Basil Blackwell, Oxford, 1983, pp. 108-126.

TsitsipIs, L. 1983. "Language shift among the Albanian speakers of Greece", AnL, 25, 288-308. 
. 